\title{
Penetrating head injuries in children due to $B B$ and pellet guns: a poorly recognized public health risk
}

\author{
Ravi Kumar, MD, ${ }^{1}$ Ramesh Kumar, MD, ${ }^{2}$ Grant W. Mallory, MD, Jeffrey T. Jacob, MD, ${ }^{1}$ \\ David J. Daniels, MD, PhD, ${ }^{1}$ Nicholas M. Wetjen, MD, ${ }^{1}$ Andrew B. Foy, MD, ${ }^{3}$ Brent R. O'Neill, MD, ${ }^{2}$ \\ and Michelle J. Clarke, MD ${ }^{1}$ \\ 'Department of Neurosurgery, Mayo Clinic, Rochester, Minnesota; '2Department of Neurosurgery, Children's Hospital Colorado, \\ Aurora, Colorado; and '3epartment of Neurosurgery, Children's Hospital of Wisconsin, Milwaukee, Wisconsin
}

OBJECTIVE Nonpowder guns, defined as spring- or gas-powered BB or pellet guns, can be dangerous weapons that are often marketed to children. In recent decades, advances in compressed-gas technology have led to a significant increase in the power and muzzle velocity of these weapons. The risk of intracranial injury in children due to nonpowder weapons is poorly documented.

METHODS A retrospective review was conducted at 3 institutions studying children 16 years or younger who had intracranial injuries secondary to nonpowder guns.

RESULTS The authors reviewed 14 cases of intracranial injury in children from 3 institutions. Eleven (79\%) of the 14 children were injured by BB guns, while $3(21 \%)$ were injured by pellet guns. In $10(71 \%)$ children, the injury was accidental. There was 1 recognized assault, but there were no suicide attempts; in the remaining 3 patients, the intention was indeterminate. There were no mortalities among the patients in this series. Ten (71\%) of the children required operative intervention, and 6 (43\%) were left with permanent neurological injuries, including epilepsy, cognitive deficits, hydrocephalus, diplopia, visual field cut, and blindness.

CONCLUSIONS Nonpowder guns are weapons with the ability to penetrate a child's skull and brain. Awareness should be raised among parents, children, and policy makers as to the risk posed by these weapons.

http://thejns.org/doi/abs/10.3171/2015.6.PEDS15148

KEY WORDS BB gun; pellet gun; air gun; air rifle; gunshot; head injury; brain injury; children; trauma

$\mathrm{N}$ ONPOWDER guns, also known as spring- or gas-powered pellet or BB guns, have long been considered children's toys. BBs are small ball bearings (Fig. $1 \mathrm{~A})$, and pellets are asymmetrical directional projectiles (Fig. 1B). Both types of ammunition can be fired from a spring-powered or compressed-gas gun. Nonpowder guns have been romanticized in the modern media, and their distribution has been unregulated at the national level. While their ability to injure soft tissues, including the eye, is well recognized, ${ }^{1}$ this risk has not deterred the propagation of these toys among children. Popular movies, such as A Christmas Story, portray the desirability of these toys among children, and this desirability remains true today: one study estimated that approximately 3.2 million $\mathrm{BB}$ and/or pellet guns are sold in the US every year. ${ }^{13}$ Based on a survey of fifth, sixth, and seventh graders, 1 in 3 chil- dren own a nonpowder gun. ${ }^{3}$ Another study found that $6 \%$ of families in the Chicago area with at least 1 child 3 years old or younger owned an air gun. ${ }^{17}$

Given the high prevalence of these weapons, it is not surprising that injuries are reported. The National Electronic Injury Surveillance System estimates that in 2013, $16,259 \mathrm{BB}$ or pellet gun injuries (code 1237) occurred in the US. An estimated 1237 of these cases were injuries to the head. Of the 16,259 nonpowder gun injuries in 2013, approximately $63 \%(10,286)$ occurred in victims 18 years old and younger. Based on a study of 101 pediatric $(<19$ years old) patients with nonpowder gun injuries, $26 \%$ of these patients had injuries caused by pellet guns, and $74 \%$ had injuries caused by BB guns. In this same study, $71 \%$ of the shootings were identified as unintentional, with assault and suicide playing smaller roles. ${ }^{1}$

ABBREVIATIONS ASTM = American Society for Testing and Materials; ATF = Bureau of Alcohol, Tobacco, Firearms, and Explosives; CPSC $=$ Consumer Product Safety Commission; $E D$ = energy density; fps = feet per second; GCS = Glasgow Coma Scale.

SUBMITTED March 13, 2015. ACCEPTED June 11, 2015.

INCLUDE WHEN CITING Published online October 23, 2015; DOI: 10.3171/2015.6.PEDS15148. 


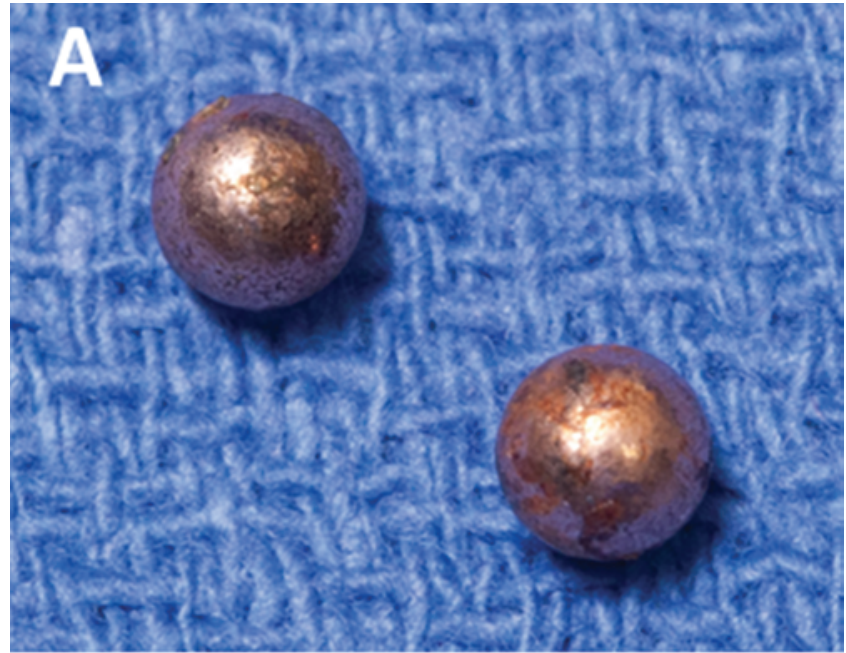

D

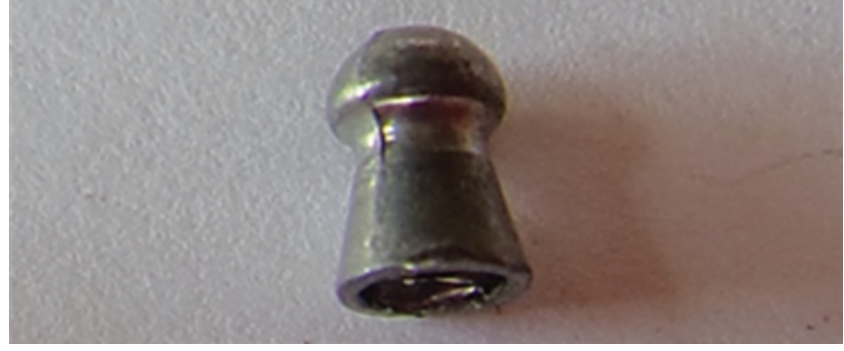

FIG. 1. A: Photograph showing 0.177-caliber copper-coated steel BBs removed from a patient's scalp. B: Photograph showing a 0.177-caliber lead pellet with a diabolo-style shape to prevent the pellet from yawing. Figure is available in color online only.

While the popularity of nonpowder guns persists, the public's appreciation of their destructive capabilities may be lacking. While the spring-loaded Red Ryder BB gun in A Christmas Story commonly evoked the adult response, "You'll shoot your eye out, kid," recent advances in compressed-gas technology have led to muzzle velocities exceeding those of regulated small-caliber weapons, ${ }^{1}$ and there have been numerous reports of intracranial injury due to these devices. ${ }^{1,2,5,9,12}$ We know that permanent neurological injury and death can be caused by this type of penetrating trauma. ${ }^{1,9}$ The increased power of nonpowder guns has made the skull a "breachable" barrier and has brought the fragile tissues of the brain into the realm of pellet and BB gun injury. This paper seeks to learn more about the injury pattern and severity of nonpowder weapon injuries in children. Further, we seek to educate providers on the increased power of these "toy" weapons and explore the social context of overriding regulation and ease of obtaining them.

\section{Methods}

We performed a retrospective review of intracranial injuries secondary to nonpowder guns at 3 institutions. Study approval was granted from the respective institutional review boards at each institution. The study popula- tion consisted of children 16 years or younger who were treated for intracranial injuries secondary to nonpowder guns. Exclusion criteria included 1) patients age 17 years or older and 2) intracranial BBs or pellets projected from gunpowder-based firearms such as handguns, shotguns, and other weapons regulated by the Bureau of Alcohol, Tobacco, Firearms, and Explosives (ATF). Cases were accrued from Mayo Clinic, Rochester Campus, from 1997 to 2014, Children's Hospital of Wisconsin from 2003 to 2014, and Children's Hospital Colorado from 2003 to 2012.

A nonpowder gun was defined as any compressed-gas or spring-powered gun. Hospital records at all 3 institutions were searched with the key words "BB" and "pellet," and cases were identified based on manual review of the records. Collected data included demographic information (patient age and sex); information surrounding the injury episode (type of weapon and intent of shooting); presenting neurological status (Glasgow Coma Scale [GCS] score and neurological examination); radiographic information (CT and CT-angiogram findings); treatment (operative procedures and rehabilitation needs); and final outcome (neurological status and disposition).

\section{Results}

Our study identified 14 pediatric patients from 3 institutions. The Mayo Clinic treated 3 patients, Children's Hospital of Wisconsin treated 5 patients, and Children's Hospital Colorado treated 6 patients. Eleven (79\%) of the 14 patients had injuries caused by BB guns and 3 (21\%) from pellet guns. Twelve (86\%) patients were male, and the average age was 8 years (range $2-15$ years). Thirteen (93\%) children were shot by another person, while 1 child accidently shot himself. Ten (71\%) of the 14 shootings were accidental, $1(7 \%)$ was an assault, and the intention was uncertain in the remaining cases. The 1 assault was a child shot by an adolescent bully. Evidence of attempted suicide was not noted (Table 1).

The skull entry site was frontal in $8(57 \%)$ patients, temporal in $2(14 \%)$, orbital in $3(21 \%)$, and parietal in $1(7 \%)$. Injury types overlapped among many patients. Injuries included subarachnoid hemorrhage in $7(50 \%)$ patients, depressed skull fracture in 4 (29\%), parenchymal contusion in $4(29 \%)$, cerebral edema in $3(21 \%)$, intraparenchymal hemorrhage in $2(21 \%)$, subdural hematoma in $1(7 \%)$, intraventricular hemorrhage in $1(7 \%)$, and pseudoaneurysm formation in $1(7 \%)$. Ten $(71 \%)$ of the 14 patients required an operative intervention. Operative interventions included craniotomy in 7 (50\%) patients, removal of the missile in $6(43 \%)$, elevation of depressed bone fragment in $2(14 \%)$, bifrontal craniectomy in $1(7 \%)$, orbital washout and exploration in $1(7 \%)$, cranioplasty in $1(7 \%)$, coiling of aneurysm in $1(7 \%)$, external ventricular drain placement in $1(7 \%)$, and ventriculoperitoneal shunt placement in 1 (7\%; Table 2).

Six (43\%) of the 14 patients had permanent neurological injuries. This included epilepsy, cognitive deficits, hydrocephalus, diplopia, visual field cut, and blindness-all occurring in separate patients. Case 3 (Table 2) developed epilepsy after being shot 12 years earlier by his brother. The injury was not brought to the attention of his parents 
TABLE 1. Overall patient characteristics and demographics

\begin{tabular}{lc}
\hline \multicolumn{1}{c}{ Patient Characteristics } & No. $(\%)$ \\
\hline Total no. of patients & 14 \\
\hline Mean age in yrs & $8.9($ range 2-15) \\
\hline Male sex & $12(86 \%)$ \\
\hline Type of weapon & \\
BB gun & $11(79 \%)$ \\
Pellet gun & $3(21 \%)$ \\
\hline Intention & \\
Accidental & $10(71 \%)$ \\
Intentional & $1(7 \%)$ \\
Unknown & $3(21 \%)$ \\
\hline Skull entry site & \\
Frontal & $8(57 \%)$ \\
Temporal & $2(14 \%)$ \\
Orbital & $3(21 \%)$ \\
Parietal & $1(7 \%)$ \\
\hline Injury & \\
Subarachnoid hemorrhage & $7(50 \%)$ \\
Depressed skull fracture & $4(29 \%)$ \\
Parenchymal contusion & $4(29 \%)$ \\
Cerebral edema & $3(21 \%)$ \\
Intraparenchymal hemorrhage & $2(21 \%)$ \\
Subdural hematoma & $1(7 \%)$ \\
Intraventricular hemorrhage & $1(7 \%)$ \\
Pseudoaneurysm & $1(7 \%)$ \\
\hline Operative intervention & $10(71 \%)$ \\
Lasting neurological deficit & $6(43 \%)$ \\
\hline
\end{tabular}

at that time. Twelve years after the injury, the patient developed a generalized seizure disorder. A CT scan revealed the presence of a right frontal $\mathrm{BB}$, and an electroencephalogram (EEG) demonstrated right frontotemporal sharp waves and temporal intermittent rhythmic delta activity. The BB was removed surgically to facilitate MRI, and the connection between his intracranial injury and seizure disorder is conjecture. The patient was lost to follow-up. Another child (Case 13) suffered optic nerve avulsion from the BB resulting in right-eye blindness. Four of the patients in this study had bihemispheric injury, of which 3 suffered permanent neurological sequelae. Overall, there were no mortalities in this study, and all children regained functional neurological status despite their injuries.

\section{Illustrative Cases \\ Case 2}

An 11-year-old male was loading BBs into a pumpaction air rifle by rolling the BBs down the barrel of the rifle (Table 2). His friend was pumping the weapon when it misfired, hitting the child. The child fell back but did not lose consciousness. A single circular entry site was noted in the center of his forehead. The patient scored 15 on the GCS and was neurologically intact upon admission to the local emergency department. A CT scan revealed 2 extracranial BBs and 4 intracranial BBs with intracranial air and no evidence of hemorrhage (Fig. 2). A CT angiogram was also performed, which revealed no evidence of vascular injury. Given the patient's excellent neurological status he was observed overnight, and the following day the 2 superficial BBs under the scalp were removed surgically for cosmetic reasons per the parents' request. It was decided not to perform a craniotomy to remove the remaining intracranial BBs. The patient was discharged on hospital

TABLE 2. Case characteristics

\begin{tabular}{|c|c|c|c|c|c|}
\hline $\begin{array}{l}\text { Case } \\
\text { No. }\end{array}$ & $\begin{array}{l}\text { Age } \\
\text { (yrs) }\end{array}$ & Region of Entry & Bihemispheric & Specific Operative Procedure & Outcome \& Final Follow-Up \\
\hline 1 & 8 & Rt parietal & & Elevate depressed skull fracture, debridement & Neurologically intact \\
\hline 2 & 11 & Rt frontal & & Removal of extracranial pellets & $\begin{array}{l}\text { Neurologically intact (subjective prob- } \\
\text { lems w/ concentration \& irritability) }\end{array}$ \\
\hline 3 & 7 & Lt frontal & Yes & Removal of BB 12 yrs after injury & Epilepsy \\
\hline 4 & 7 & Rt frontal & & Craniotomy for removal of pellet & Neurologically intact \\
\hline 5 & 15 & Lt frontal & & Craniotomy for removal of pellet & Neurologically intact \\
\hline 6 & 12 & Rt frontal & & None & Trouble in school \\
\hline 7 & 2 & Rt frontal & & Craniotomy for removal of pellet, elevate skull fracture & Neurologically intact \\
\hline 8 & 13 & Lt medial epicanthus & & None & Neurologically intact \\
\hline 9 & 11 & Rt frontal & Yes & Craniotomy \& removal of $\mathrm{BB}$ & No deficits \\
\hline 10 & 9 & Lt frontal & Yes & $\begin{array}{l}\text { Bifrontal craniectomy, coiling of pseudoaneurysm, } \\
\text { coiling of residual aneurysm, autologous cranio- } \\
\text { plasty \& EVD placement, synthetic cranioplasty \& } \\
\text { VP shunt placement }\end{array}$ & $\begin{array}{l}\text { Hydrocephalus, required rehab, returned } \\
\text { close to baseline }\end{array}$ \\
\hline 11 & 8 & Lt temporal & Yes & None & Persistent diplopia \\
\hline 12 & 12 & Lt orbit & & Orbital washout \& exploration & Lt inferior temporal field cut \\
\hline 13 & 6 & Rt orbit & & Rt laser retinopexy/globe exploration & Blind in rt eye \\
\hline 14 & 3 & Lt temporal & & None & Neurologically intact \\
\hline
\end{tabular}

$E V D=$ external ventricular drain; $V P=$ ventriculoperitoneal. 

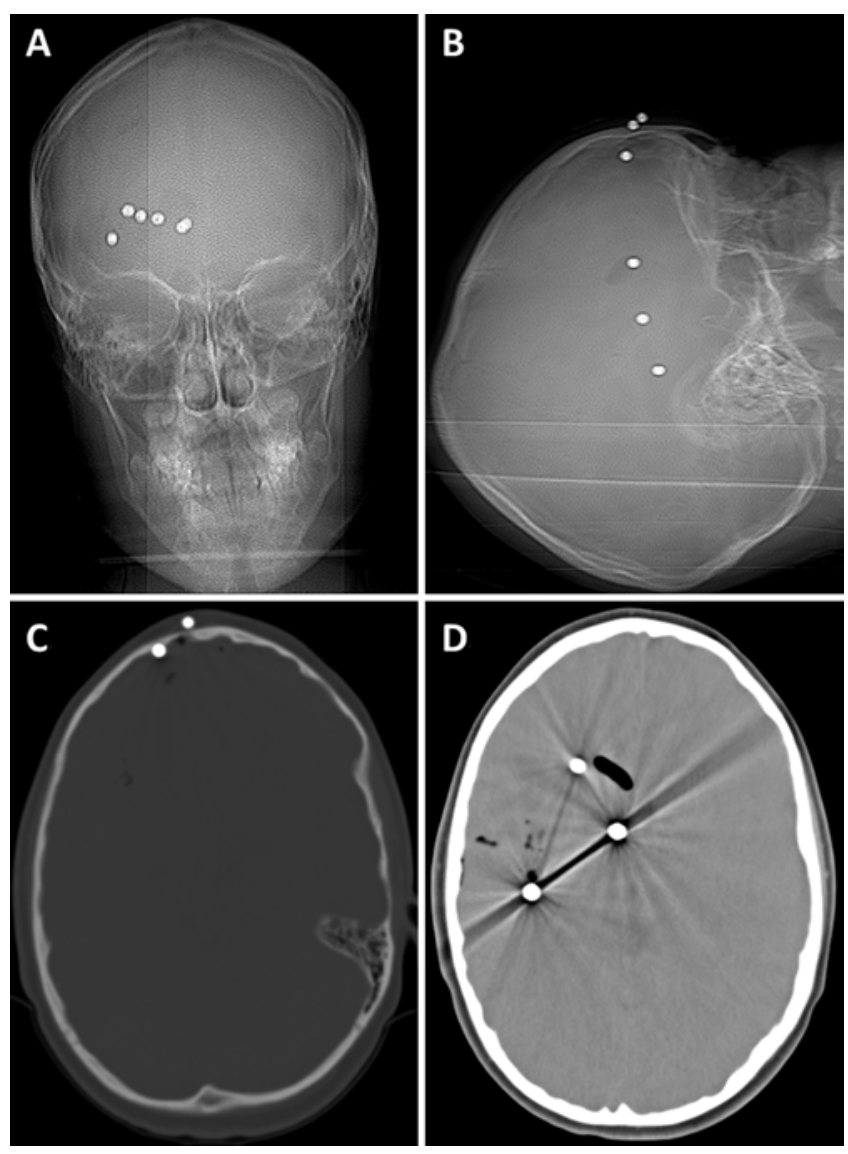

FIG. 2. Case 2. Anteroposterior (A) and lateral (B) radiographs showing the patient's skull with 6 embedded BBs: 4 intracranial and 2 extracranial. C: Axial CT image showing the same patient's head with bone windowing. There is a small breach in the frontal bone where all $4 \mathrm{BBs}$ entered. D: Axial CT image with brain windowing showing 3 of the intracranial BBs. There is intraparenchymal and intraventricular air.

Day 3. A CT scan 2 months later revealed no migration of the retained BBs. Two months following his injury, the patient's mother noted that since the injury the patient had persistent irritability and an inability to concentrate. The patient was followed for 8 months with no evidence of infection, seizure activity, or migration of the BBs on subsequent scans. The patient was advised to follow-up on an as-needed basis.

\section{Case 10}

A 9-year-old male was accidentally shot in the left temporal region by his brother while both were playing with a BB gun. He fell to the ground immediately and was noted to be confused and somnolent at the scene. Soon after presentation he began to decline, neurologically requiring emergent intubation. An initial CT scan of the head showed significant bifrontal cerebral edema, intraparenchymal hemorrhage within the BB tract, and subarachnoid hemorrhage within the basilar cisterns (Fig. 3A). CT angiogram showed that the BB was adjacent to the right $\mathrm{M} 1$ branch of the middle cerebral artery. The patient underwent an emergent bifrontal decompressive craniectomy. Several days later, a cerebral angiogram showed a new pseudoaneurysm of the proximal left A2 segment of the anterior cerebral artery (Fig. 3B) and moderate-tosevere vasospasm of the bilateral anterior and middle cerebral artery circulations. He underwent coil embolization of the A2-segment pseudoaneurysm and multiple serial angiograms with angioplasty and intraarterial injection of verapamil for symptomatic vasospasm. Given the progressive enlargement of the A2 pseudoaneurysm (Fig. 3C), a second coil embolization was performed 1 week after the initial treatment. The patient's neurological function slowly improved, and he was eventually transferred to a rehabilitation service. Approximately 3 months after his initial presentation, he underwent autologous cranioplasty. Over 3 months, he developed persistent subgaleal fluid collections and a significant amount of autologous flap resorption was noted. The remaining portion of the autologous graft was removed and replaced with a synthetic allograft cranioplasty and ventriculoperitoneal shunt placement. The patient has made a nearly full recovery with no grossly apparent neurological dysfunction.

\section{Results of Market Survey}

In addition to exploring case data, the authors searched a popular online retailer (www.amazon.com) with the key word "BB gun" and sorted the results by "new and best sellers." Airsoft guns that shoot a soft plastic pellet were excluded. Of the top 10 results, the average price was \$66.98 (range \$29.99-\$153.84) and the average muzzle velocity was 750 feet per second (fps; range 350-1250 fps). None of the weapons in this category had markers to designate them as nonpowder guns such as blaze orange or highly visible colors. This demonstrates how accessible and affordable high-powered nonpowder weapons can be.

\section{Discussion}

$\mathrm{BB}$ and pellet guns are common childhood possessions with unrecognized dangers. In our study of 3 institutions, we found that these toys can result in intracranial injuries and serious long-term neurological sequelae in some patients. Most cases of intracranial nonpowder weapon injuries occurred accidentally, with entry wounds most commonly found in the frontal and temporal regions. This study demonstrates the vulnerability of childrens' brains to modern $\mathrm{BB}$ and pellet guns.

$\mathrm{BB}$ guns have evolved to become more powerful, and subsequently more dangerous. Nonpowder guns were traditionally spring-loaded weapons that would fire a metal or plastic projectile at less than $350 \mathrm{fps}$, until 25 years ago when air-powered weapons became readily available. ${ }^{11}$ Recent advances in compressed-gas technology have increased muzzle velocities of nonpowder guns to 9001200 fps. ${ }^{1}$ In comparison, a 0.22-caliber rifle has a muzzle velocity of around $1000 \mathrm{fps}$.

In general, projectiles damage tissue by crush and temporary cavitation.7 Given the BB and pellet's small mass and dimensions, temporary cavitation is not a significant cause of morbidity in brain injury. Instead, BBs and pellets crush the tissue they penetrate, creating a permanent cavity. This cavity can cause significant injury if it traverses eloquent portions of the brain or vascular structures.

The physics behind BB guns underscores their dangerous ability to cause intracranial injuries. The ability of a 

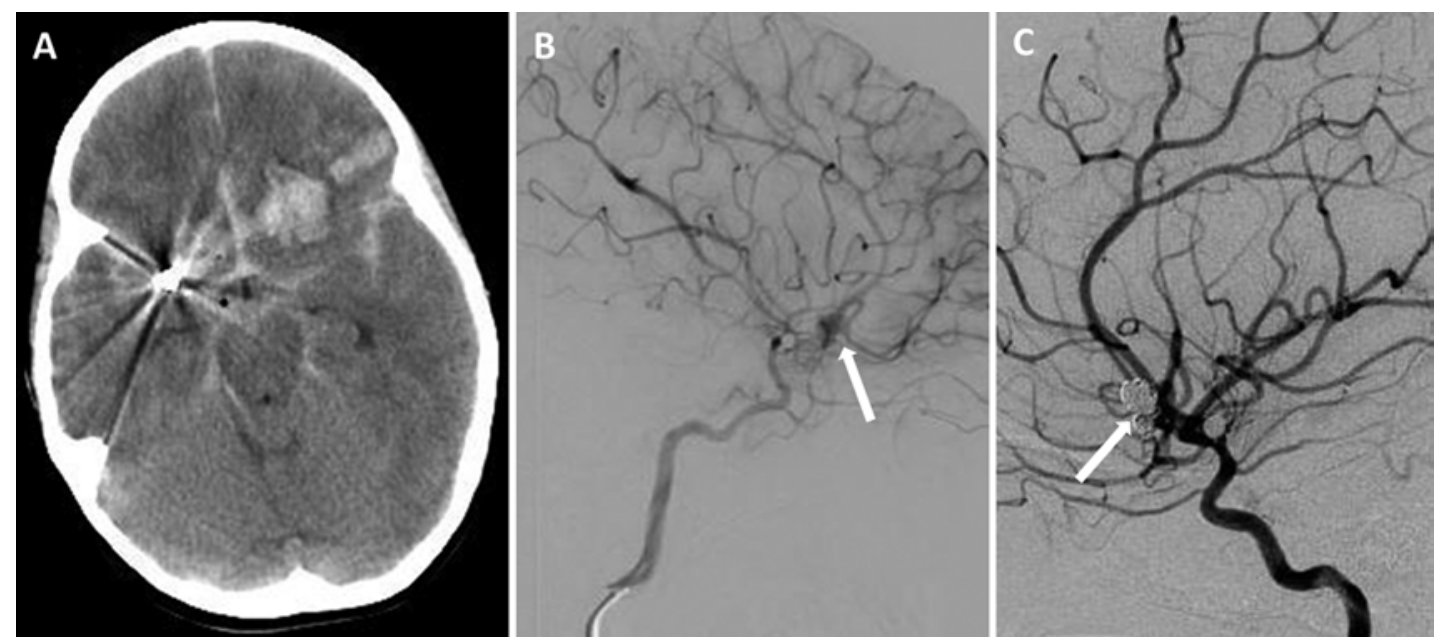

FIG. 3. Case 10. A: Axial CT image of the patient's head with brain windowing. The BB entered the left temporal area and traversed along the inferior, bilateral frontal lobes to rest near the right sylvian fissure. There is a significant amount of bifrontal cerebral edema, intraparenchymal hemorrhage within the BB tract, and subarachnoid hemorrhage within the basilar cisterns. B: Digital subtraction cerebral angiogram with injection of the left internal carotid artery. A pseudoaneurysm can be seen arising from the A2 segment of the left anterior cerebral artery (arrow). C: Digital subtraction cerebral angiogram with injection of the left internal carotid artery showing subsequent coiling of the pseudoaneurysm (arrow).

$\mathrm{BB}$ or pellet to penetrate the skull is determined by its energy density (ED). This is represented by the equation ED $=\mathrm{E} / \mathrm{A}$ (kinetic energy/cross-sectional area of the bullet), where kinetic energy equals (mass $\times$ velocity $\left.{ }^{2}\right) / 2$. Based on a previous study, the minimum $\mathrm{ED}$ of a BB to penetrate an adult skull is $7 \times 10^{4} \mathrm{ft} \cdot \mathrm{lbs} / \mathrm{sq} \mathrm{ft} .^{18}$ The most common caliber of a BB or pellet is 0.177 , which equals a 0.177 -inch diameter. BB and pellet weights range from 5.1 to 16.1 grains, where 437.5 grains equals $28.35 \mathrm{~g}$. Using the lowest BB weight (5.1 grains or $0.33 \mathrm{~g}$ ), adult skull penetration can occur at $1025.9 \mathrm{ft} / \mathrm{sec}$. If we consider a common pellet weight of 7.9 grains or $0.5 \mathrm{~g}$, the adult skull penetration velocity reduces to $825.1 \mathrm{ft} / \mathrm{sec}$ (Table 3). As we discovered in our simple retail survey, easily available modern nonpowder guns have muzzle velocities ranging up to 1250 $\mathrm{ft} / \mathrm{sec}$. Thus, today's modern compressed-gas-powered weapons generate enough muzzle velocity to penetrate an adult skull.

Theoretically, children are at an even greater risk of intracranial injury. Pediatric patients have varying degrees of skull development and thickness as well as thinner overlying soft tissue ${ }^{6,10}$ making skull penetration possible at lower velocities than would be needed in an adult cohort. Additionally, children may play in close proximity to these weapons, which will increase the velocity of the missile at impact. One study estimated the mean distance between child and gun during these injuries was 5 feet. ${ }^{1}$ As velocity decreases with distance as a result of air resistance, proximity to the gun likely has an effect on penetrative ability.

Despite the power of these weapons, there are no federal regulations pertaining to the purchase and use of nonpowder guns. Unlike powder firearms, which are overseen by the ATF, nonpowder guns fall under the jurisdiction of the Consumer Product Safety Commission (CPSC). The CPSC will regulate a consumer product that imposes substantial risk to the user, yet they have not imposed spe- cific regulations on nonpowder guns as a whole. The BB and pellet gun industry has issued a "Standard Consumer Safety Specification for Nonpowder Guns" (ASTM F589). These can be purchased for $\$ 49$ through the American Society for Testing and Materials (ASTM), limiting its visibility to consumers and healthcare professionals. Many states have filled the gaps on nonpowder gun regulation left unfilled at the federal level. Information on nonpowder

\section{TABLE 3. Energy density calculations}

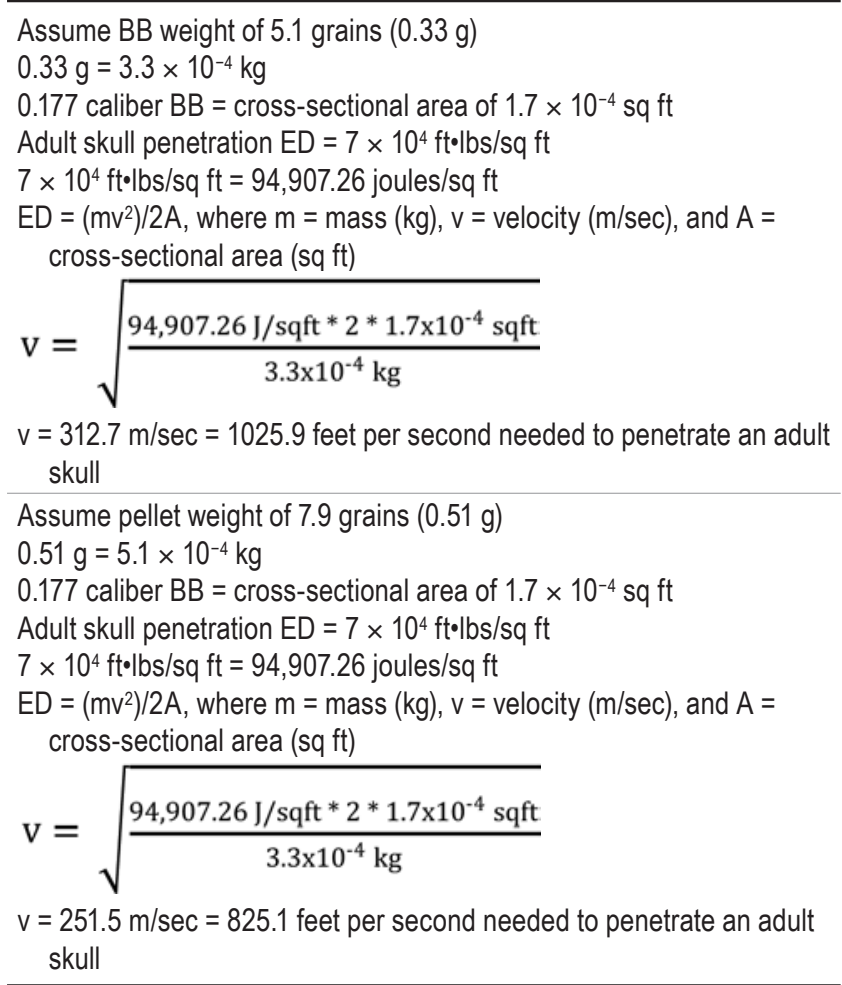


gun regulation at the state level is complex and dynamic. An excellent resource for up-to-date information on state laws and regulations is compiled by the Law Center to Prevent Gun Violence. ${ }^{8}$

In addition to the increased power of unregulated nonpowder weapons, children may be at higher risk of injury because of societal misconceptions and behavioral reasons. Adult supervision and formal weapons training may not be provided to children because these guns are often classified as toys or sporting equipment. ${ }^{4}$ As noted in Case 2 above, children may also not understand or ignore instructions for recommended use. In this case, accidental or intentional over-inflation of the air chamber by manually pumping can result in a muzzle velocity exceeding the manufacturer's specifications. While many pellet gun models can only be pumped once to charge the gun, some BB gun models allow manually pumping of the air chamber, leading to over-inflation and BB velocities greater than advertised or recommended by the manufacturer. Also illustrated in Case 2, it is possible to muzzle-load BBs to exceed the recommended $1 \mathrm{BB}$ per chamber maximum. This results in multiple BBs being fired simultaneously and greatly increases the overall mass and penetrative ability. The BBs in this case were accidently fired at close range and had the same entry site in the frontal bone. This may have led to a billiard ball-like phenomenon where the trailing BBs transferred their energy to the leading BBs. As the muzzle velocity and penetrative ability of nonpowder guns approach, and in some cases exceed, that of regulated powder-based guns, society may need to reconsider how these weapons are categorized, regulated, and supervised.

As neurosurgeons on the front lines responding to intracranial nonpowder gun injuries, we have 2 important responsibilities. First, by understanding the dangers of nonpowder gun technology, we have a duty to educate parents and children on being responsible gun owners and operate these weapons safely. Many parents, and indeed many neurosurgeons, likely consider these weapons to be toys, but they are pushing the definition of true guns. Second, we must comprehensively treat injured patients and be sure they recognize the potential long-term consequences of intracranial injuries. This begins by understanding that what may appear to be a superficial injury by a nonpowder gun must include a comprehensive trauma evaluation. Aside from the intracranial damage noted in this and other studies of nonpowder weapons, there are long-term implications of retained BBs that must be explained to parents and children. For instance, the majority of brain injuries in our study were from BBs, which are almost universally steel ball bearings coated with a noncorrosive laminate metal such as copper or zinc (Fig. 1A). These steel BBs will be strongly ferromagnetic and can move in a magnetic field, potentially causing more intracranial injury during MRI.14 The majority of pellets are "diabolo" style, with a skirt on its trailing end resembling a badminton birdie (Fig. 1B). While pellets are usually made of lead, which does not deflect in a magnetic field, some pellets have steel tips or are made of lead-free alloys that may be ferromagnetic. As with any intracranial metal fragment, a child may be precluded from MRI scanning for life, and thorough documentation of the intracranial fragment should be made.
While no cases of intracranial migration of a BB or pellet were documented in this study, retained bullet fragments have been reported to migrate..$^{15,16,19}$ Specifically, copper and lead have been implicated in this process, which are 2 of the major component metals in BBs and pellets, respectively. ${ }^{16}$ Removal of accessible intracranial or extracranial fragments should be performed whenever it is safe to do so, as this will allow the patient future access to MRI and also ensure that intracranial migration does not become an issue. Although there were no cases of infection or meningitis in our series, open and obviously contaminated wounds should be considered for debridement. In our study, 10 of 14 children required surgery.

There are several weaknesses in this retrospective observational study. Our data does not allow us to determine the true incidence or prevalence of intracranial injury secondary to nonpowder guns. Additionally, limitations and subjectivity of follow-up make it difficult to understand the long-term sequelae of these injuries. However, the destructive injuries recorded in this study and the technological advances of these weapons, which exceed public awareness, make this a public health concern. Just as we have a responsibility to counsel patients and families on the importance of helmet use, pediatric neurosurgeons responding to nonpowder weapon injuries have a responsibility to understand and share the dangers of these weapons with parents and children.

\section{Conclusions}

Nonpowder guns, otherwise known as air, BB, or pellet guns, are dangerous weapons that are marketed as children's toys. Because of advancing technology in these nonpowder guns that increases muzzle velocities, the skull is now breachable by BBs and pellets, putting the brain at risk. Children, in particular, are vulnerable to injury because of their less-developed skulls, lack of danger awareness, and their gravitation toward these child-marketed weapons.

\section{Acknowledgment}

We would like to thank Pili McComas for providing Fig. 1B.

\section{References}

1. Bratton SL, Dowd MD, Brogan TV, Hegenbarth MA: Serious and fatal air gun injuries: more than meets the eye. Pediatrics 100:609-612, 1997

2. Chhetri DK, Shapiro NL: A case of a BB-gun pellet injury to the ethmoid sinus in a child. Ear Nose Throat J 83:176, 178, 180,2004

3. Cunningham PB, Henggeler SW, Limber SP, Melton GB, Nation MA: Patterns and correlates of gun ownership among nonmetropolitan and rural middle school students. J Clin Child Psychol 29:432-442, 2000

4. Damore DT, Ramundo ML, Hanna JP, Dayan PS: Parental attitudes toward BB and pellet guns. Clin Pediatr (Phila) 39:281-284, 2000

5. Ford EG, Senac MO Jr, McGrath N: It may be more significant than you think: BB air rifle injury to a child's head. Pediatr Emerg Care 6:278-279, 1990

6. Garfin SR, Roux R, Botte MJ, Centeno R, Woo SL: Skull osteology as it affects halo pin placement in children. J Pediatr Orthop 6:434-436, 1986 
7. Hollerman JJ: Wound ballistics is a model of the pathophysiology of all blunt and penetrating trauma. Emerg Radiol 5:279-288, 1998

8. Law Center To Prevent Gun Violence: Non-Powder Guns Policy Summary. (http://smartgunlaws.org/non-powderguns-policy-summary/) [Accessed August 7, 2015]

9. Lawrence HS: Fatal nonpowder firearm wounds: case report and review of the literature. Pediatrics 85:177-181, 1990

10. Margulies SS, Thibault KL: Infant skull and suture properties: measurements and implications for mechanisms of pediatric brain injury. J Biomech Eng 122:364-371, 2000

11. McNeill AM, Annest JL: The ongoing hazard of BB and pellet gun-related injuries in the United States. Ann Emerg Med 26:187-194, 1995

12. Miner ME, Cabrera JA, Ford E, Ewing-Cobbs L, Amling J: Intracranial penetration due to BB air rifle injuries. Neurosurgery 19:952-954, 1986

13. Nguyen MH, Annest JL, Mercy JA, Ryan GW, Fingerhut LA: Trends in BB/pellet gun injuries in children and teenagers in the United States, 1985-99. Inj Prev 8:185-191, 2002

14. Oliver C, Kabala J: Air gun pellet injuries: the safety of MR imaging. Clin Radiol 52:299-300, 1997

15. Rammo RA, DeFazio MV, Bullock MR: Management of migrating intracranial bullets: lessons learned from surviving an AK-47 bullet through the lateral brainstem. World Neurosurg 77:591.e19-591.e24, 2012

16. Rapp LG, Arce CA, McKenzie R, Darmody WR, Guyot DR, Michael DB: Incidence of intracranial bullet fragment migration. Neurol Res 21:475-480, 1999

17. Senturia YD, Binns HJ, Christoffel KK, Tanz RR: In-office survey of children's hazard exposure in the Chicago area: age-specific exposure information and methodological lessons. J Dev Behav Pediatr 14:169-175, 1993

18. Sights WP Jr: Ballistic analysis of shotgun injuries to the central nervous system. J Neurosurg 31:25-33, 1969

19. Zafonte RD, Watanabe T, Mann NR: Moving bullet syndrome: a complication of penetrating head injury. Arch Phys Med Rehabil 79:1469-1472, 1998

\section{Disclosure}

The authors report no conflict of interest concerning the materials or methods used in this study or the findings specified in this paper.

\section{Author Contributions}

Conception and design: Ravi Kumar, Ramesh Kumar, Foy, O'Neill, Clarke. Acquisition of data: Ravi Kumar, Ramesh Kumar, Mallory, Jacob, Daniels, Wetjen, O’Neill, Clarke. Analysis and interpretation of data: all authors. Drafting the article: Ravi Kumar, Ramesh Kumar, O’Neill, Clarke. Critically revising the article: all authors. Reviewed submitted version of manuscript: Ravi Kumar, Ramesh Kumar, Mallory, Jacob, Daniels, O'Neill, Clarke. Approved the final version of the manuscript on behalf of all authors: Ravi Kumar. Administrative/technical/material support: Clarke. Study supervision: Clarke.

\section{Correspondence}

Ravi Kumar, Department of Neurosurgery, Mayo Clinic, 200 1st St. SW, Rochester, MN 55905. email: kumar.ravi@mayo.edu. 